\title{
NOVO CORONAVÍRUS: A LINHA DE FRENTE NO COMBATE À PANDEMIA
}

\author{
NEW CORONAVIRUS: THE FRONTLINE IN \\ THE FIGHT AGAINST THE PANDEMIC
}

\author{
Ana Luiza Ferreira Aydogdu ${ }^{1}$
}

\section{RESUMO}

Introdução: A doença que surgiu na China no final de dezembro de 2019 e mais tarde ficou conhecida como COVID-19 vem causando tragédias ao redor do mundo. Na linha de frente ao combate à pandemia estão os profissionais da saúde, que constantemente arriscam suas vidas. Objetivo: Refletir sobre o contexto institucional dos profissionais da saúde que atuam como linha de frente no combate à pandemia do novo coronavírus. Método: Reflexão teórica baseada na literatura nacional e internacional sobre profissionais da saúde que atuam como linha de frente no combate à pandemia do novo coronavírus. Resultados: Profissionais da saúde foram decisivos nas epidemias e pandemias que ameaçaram a humanidade. Sempre correram riscos ao realizarem suas funções. Com o surgimento da pandemia do novo coronavírus os riscos aumentaram. Entre os profissionais da saúde, são vários infectados pela COVID-19 em torno do mundo, para alguns, o contágio foi fatal. Profissionais da saúde precisam ter acesso a equipamentos de proteção individual em quantidade e qualidade adequadas, além de receberem treinamento para utilizá-los. Esses profissionais estão adoecendo física e psicologicamente, o que ameaça ainda mais a população mundial. Conclusões: Para continuarem realizando suas funções da melhor forma possível, visando controlar a pandemia do novo coronavírus, profissionais da saúde precisam ser protegidos e apoiados pelo governo, pela instituição onde trabalham e também pela população.

Palavras-chave: Doenças Transmissíveis, Infecções por Coronavírus, Pandemias, Pessoal de Saúde.

\section{ABSTRACT}

Introduction: The disease which appeared in China in late December 2019 and later became known as COVID-19 has been causing tragedies around the world. On the front line against the pandemic are healthcare workers, constantly risking their lives. Objective: To reflect on the institutional context of healthcare workers who act as frontline in combating the pandemic of the new coronavirus. Method: Theoretical reflexion, based on national and international literature on healthcare workers who act as frontline in fighting the new coronavirus pandemic. Results: Healthcare workers were decisive in the epidemics and pandemics that threatened humanity. They have always taken risks when carrying out their duties. With the emergence of the new coronavirus pandemic, the risks increased. Several healthcare workers were infected with COVID-19 around the world, for some of them the disease was fatal. Healthcare workers need to have access to personal protective equipment in adequate quantity and quality; they need also to be trained to use it. These professionals are becoming physically and psychologically ill, which further threatens the world's population. Conclusions: Healthcare workers need to be protected and supported by the government, by the institution where they work and also by the population, so they can continue to perform their duties in the best way in order to control the pandemic of the new coronavirus.

Keywords: Communicable Diseases, Coronavirus Infections, Health Personnel, Pandemics.

\footnotetext{
${ }^{1}$ Enfermeira. Mestra em Administração Hospitalar e de Instituições de Saúde. Doutoranda em Administração em Enfermagem pela Faculdade de Enfermagem Florence Nightingale da Universidade de Istambul - Cerrahpasa. Turquia. E-mail: luizafl@gmail.com
} 


\section{INTRODUÇÃO}

A COVID-19 surgiu na China no final de 2019 (WHO, 2020a) e em poucos meses se tornou uma pandemia. Hoje, dia 09 de maio de 2020 está presente em 187 países, totalizando 3.954.246 casos e 275.160 óbitos (JOHNS HOPINKS UNIVERSITY, 2020). Na mesma data, no Brasil são 155.939 casos e 10.627 óbitos (BRASIL, 2020a).

Na linha de frente ao combate à doença estão os profissionais da saúde, que contabilizam mais de 59 milhões de pessoas no mundo (JOSEPH; JOSEPH, 2016), aproximadamente 3,5 milhões deles atuam no território nacional (FIOCUZ, 2015; IBGE, 2018). Cerca de 90 mil profissionais da saúde foram infectados pelo novo coronavírus (SARS-CoV-2) em torno do mundo (ICN, 2020). No Brasil, mais de 8 mil profissionais da saúde apresentaram sintomas da doença (FOLHAPRESS, 2020).

A quantidade de profissionais da saúde em alguns países já era insuficiente mesmo antes da pandemia de COVID-19 (OECD, 2019) e suas condições de trabalho já apresentavam muitos pontos negativos, como muitas horas de plantão, baixos salários e falta de material necessário para realização de suas funções (DIKMEN et al., 2014; MELGAREJO; BALLEN, 2010; NDEJJO et al., 2015; YAZICI; ELBAS, 2017). Com o surgimento do SARS-CoV-2 a situação piorou. Os profissionais da saúde enfrentam novos problemas como medo de contrair a doença e infectar entes queridos, falta de Equipamentos de Proteção Individual (EPIs), escassez de funcionários pelo afastamento de colegas devido à doença, entre outros (ADAMS; WALLS, 2020; LANCET, 2020; WHO, 2020b; SCHWARTZ; KING; YEN, 2020; SOUZA E SOUZA; SOUZA, 2020).

Os profissionais da saúde sempre tiveram papéis marcantes nas tragédias que ameaçaram a humanidade através dos tempos. Durante epidemias e pandemias muitos estiveram sob riscos constantes e alguns perderam a vida (JI et al., 2017; REZENDE, 2019; SEPKOWITZ; EISENBERG, 2005).

Em muitos países como Espanha, Itália, Turquia, Estados Unidos, Argentina e também no Brasil podemos acompanhar diariamente notícias de profissionais da saúde que foram afastados do trabalho devido à COVID-19 (CHA, 2020; GONZAGA, 2020; NUGENT, 2020; REGULY, 2020; SATUR, 2020; ULU, 2020). Sem um número adequado de funcionários capacitados nos estabelecimentos de saúde, o controle da pandemia é totalmente impossível.

Os governos de diversos países apresentaram estratégias para resolver o problema, como novas contratações, impedimento de demissões e trabalho voluntário (BRASIL, 2020b; 2020c; PORTUGAL, 2020; TURQUIA, 2020). Entretanto, se medidas de prevenção não forem tomadas de forma correta, o adoecimento e a morte de profissionais da saúde continuarão aumentando, o que além de ser traumático em termos emocionais, levará à estagnação do sistema de saúde em vários países, inclusive no Brasil (SOUZA E SOUZA; SOUZA, 2020).

O objetivo desse artigo é refletir sobre o contexto institucional dos profissionais da saúde que atuam como linha de frente no combate à pandemia do novo coronavírus. 


\section{MÉTODO}

Trata-se de um reflexão teórica baseada na leitura e interpretação de artigos científicos, relatórios e notícias sobre o contexto institucional dos profissionais da saúde que atuam como linha de frente no combate à pandemia do novo coronavírus.

A coleta de informações foi realizada no período de abril e maio de 2020 nas seguintes bases de dados: Pubmed, Literatura Latino-Americana e do Caribe em Ciências da Saúde (LILACS) e Google Acadêmico utilizando os descritores: "COVID-19", "profissionais da saúde" e "pandemia".

Em busca de notícias sobre o assunto em diferentes países foi feita uma pesquisa no google utilizando as mesmas palavras-chave acima mencionadas.

Realizou-se ainda consultas a sites institucionais nacionais e internacionais visando acessar relatórios e reportagens sobre o assunto.

Procurou-se discutir estudos que abrangessem o tema COVID-19 e profissionais da saúde.

\section{RESULTADOS E DISCUSSÃO}

Médicos, enfermeiros, técnicos de enfermagem, atendentes, fisioterapeutas, entre outros estão na linha de frente nos estabelecimentos de saúde lutando contra o SARS-CoV-2. As funções desenvolvidas por esses profissionais levam ao desgaste físico e emocional.

O trabalho do profissional da saúde, sempre foi caracterizado por longas horas de plantões, onde sua saúde física e psicológica estão sob riscos constantes (CARLESI, et al., 2017; DALRI; ROBAZZI; SILVA, 2010; FERNANDES et al.,2013; MELGAREJO; BALLEN, 2010; SANGOI; GAHLEN; STOBÄUS, 2016; ZHOU et al., 2017).

Em muitos países como Índia, México, Colômbia, China, Costa Rica, Turquia, Brasil entre outros, o número de médicos e enfermeiros está abaixo da média dos países que compõem a Organização de Cooperação e de Desenvolvimento Econômico (OCDE). No Brasil, são 0,8 médicos e 1,5 enfermeiros para cada 1.000 habitantes, enquanto a média da OCDE é de 3,5 para médicos e 8,8 para enfermeiros (OECD, 2019).

Um número escasso de profissionais aumenta os já existentes riscos ocupacionais. Profissionais acabam tendo menos horas de folga para suprir a falta de colegas, trabalhando cansados e desmotivados, o que amplia a probabilidade de cometerem erros, colocando suas próprias vidas e a dos pacientes em risco.

Profissionais da saúde enfrentam todos os dias situações com altos riscos de causar danos físicos e psicológicos, como contaminação por doenças infecto-contagiosas, exposição aos materiais radioativos e químicos, óbitos de pacientes, falta de medicamentos e de equipamentos de proteção (DALRI; ROBAZZI; SILVA, 2010; NEVES et al., 2011; NIA et al., 2016). O surgimento da COVID-19 veio 
agravar esse quadro. O SARS-CoV-2 é um vírus ainda pouco conhecido, cujo risco de contaminação é extremamente alto, aumentando assim a apreensão dos profissionais da saúde quanto à infecção e trazendo ainda uma sensação de incapacidade frente a uma doença que causa tantos óbitos (LANCET, 2020; SCHWARTZ; KING; YEN, 2020; WHO, 2020c; WHO, 2020d).

Profissionais da saúde são, sem dúvida, os mais expostos à doença, são eles que estão constantemente em contato direto com o vírus (ALICI; BEYAN; SIMSEK, 2020; HUANG; ZHAO, 2020). Enfrentam ainda dilemas durante a assistência, ao terem que decidir pela vida de pacientes e também com relação às atitudes que devem ser tomadas quando eles próprios apresentem sintomas de COVID-19. Têm que vencer ainda o medo do contágio ao prestar cuidados aos pacientes (MENON; PADLY, 2020).

Profissionais da saúde sempre foram peças-chave durante epidemias e pandemias. Não foram poucos os riscos que correram até os hoje. Além de enfrentarem discriminações e críticas ao decorrer da história. Foram também eles que trouxeram muitas medidas sanitárias, evitando o contágio e a morte de um número ainda maior de pessoas. Muitos foram os primeiros contatos de doentes, adquirindo assim a doença e vindo a óbito. Alguns deles ainda hoje convivem com sequelas e preconceitos devido a infecções adquiridas durante a realização de suas funções (GRECO; NEVES, 1993; GOULART, 2005; JI et al., 2017; VELLOSO, 2008; WYZGOWSKI et al., 2016).

No caso da COVID-19 muitos profissionais da saúde foram afastados do trabalho por apresentarem teste positivo ou sintomas da doença. A equipe de enfermagem, que representa a classe mais numerosa da área da saúde também é a que tem um número maior de infectados e de óbitos. No mundo, segundo o Conselho Internacional de Enfermeiros (ICN) aproximadamente 90 mil profissionais da saúde foram infectados e mais de 260 profissionais da enfermagem perderam suas vidas no combate ao SARS-CoV-2 (ICN, 2020). No Brasil são mais de 8 mil profissionais da saúde contaminados (FOLHAPRESS, 2020) e até a primeiros dias de maio, 98 profissionais da enfermagem tinham sido vítimas fatais da COVID-19. Com esses números o país ocupa o primeiro lugar em mortes de profissionais da enfermagem devido à doença. Segundo o Conselho Federal de Enfermagem (COFEN), no território nacional o número de óbitos entre o pessoal de enfermagem já é maior que em países como Itália e a Espanha juntos (BRASIL, 2020d; 2020e).

Faz-se necessário conhecer as razões que levam à infecção e morte de tantos profissionais da saúde, que atuam no combate à COVID-19, ampliando medidas para evitá-las.

No início da epidemia, ainda na China, muitos profissionais foram contaminados devido ao desconhecimento do agente causador da doença e de suas formas de contágio. Mais tarde o grande número de doentes facilitou a contaminação e depois houve a falta ou o uso inadequado de EPIs que levou a alta incidência da doença entre os profissionais da saúde(WANG; ZHOU; LIU, 2020).

Atualmente com as informações disponíveis sobre o vírus e as medidas protetivas, as contaminações e os óbitos dos profissionais da saúde deveriam ter seus números reduzidos. 
Entretanto, em instituições de saúde de alguns países a escassez de EPIs continua. Outro agravante é a manutenção de profissionais idosos e doentes crônicos no atendimento aos pacientes com COVID-19 (BRASIL, 2020d; GLYNN, 2020; WHO, 2020b).

Com o afastamento de tantos profissionais devido à doença os governantes ao redor do mundo procuram formas para resolver o problema de falta de funcionários. No Brasil, houve convocação de profissionais da saúde através da portaria $n^{\circ} 639$ publicada no Diário Oficial da União no dia 02 de abril de 2020. Ocorreram ainda contratações temporárias, médicos e enfermeiros voluntários partiram para outros estados. Além disso, alunos dos últimos anos de cursos como medicina, enfermagem, farmácia e fisioterapia foram chamados para atuarem contra à COVID-19 através do edital $n^{\circ} 4$ de 31 de março de 2020, que consta no Diário Oficial da União no 62 de 01 de abril de 2020 (BRASIL, 2020b; 2020c; 2020f; 2020g; RIO DE JANEIRO, 2020)

Se o profissional não estiver devidamente preparado para exercer a profissão e não for adequadamente treinado para o combate ao SARS-CoV-2, a contratação de novos funcionários, que é vista como solução para o problema pode, na verdade, trazer ainda mais riscos para o sistema de saúde. O profissional da saúde precisa ser corretamente treinado para o atendimento ao paciente com COVID-19. O funcionário deve conhecer os protocolos de atendimento para que sua conduta frente à doença seja adequada, reduzindo assim os riscos para os pacientes e para si próprio.

É preciso educar para o cuidado ao paciente com COVID-19, lembrando que o uso correto de EPIs é algo que requer conhecimento (RODRIGUES; SILVA, 2020; SCHWARTZ; KING; YEN, 2020). O treinamento de um profissional da saúde, principalmente frente a uma doença tão contagiosa é de suma importância (JI et al., 2017; RODRIGUES; SILVA, 2020) e demanda tempo. Em se tratando de serviços como Unidades de Tratamento Intensivo (UTI), onde muitos dos pacientes com COVID-19 estão internados, pode demandar muitos meses. Entretanto, a atual realidade não fornece tempo, nem oportunidade para treinar novos profissionais, aumentando assim ainda mais o risco de contágio.

Buscando suprir a falta de profissionais da saúde não é certo arriscar a vida deles próprios e de pacientes, colocando na linha de frente contra uma doença tão contagiosa, funcionários sem treinamento adequado ou que não tenham concluído seus cursos. É preciso ainda considerar a possibilidade desses profissionais não estarem psicologicamente preparados para essa tarefa, o que poderá gerar consequências irreparáveis, inclusive pôr em risco suas vidas.

Para o controle da pandemia é preciso preservar a saúde física e mental dos profissionais. Profissionais da saúde estão esgotados, é importante adaptar horários de trabalho e aumentar o número de funcionários aptos para o exercício de suas funções. Além do fornecimento em número e qualidade adequados de EPIs, o que os protegerá fisicamente. É preciso ainda cuidar da saúde mental desses profissionais (CETINTEPE; ILHAN, 2020; GALLASCH et al., 2020; GODDERIS, BOONE; BAKUSIC, 2020; RODRIGUES; SILVA, 2020; OIT, 2020). 
A COVID-19 representa uma ameaça para a saúde física dos profissionais da saúde não só devido aos riscos de contágio pelo vírus, mas também porque os casos de agressões contra esses profissionais aumentaram com o surgimento da doença (AYDOGDU, 2020). A saúde psicológica desses profissionais também foi afetada com o surgimento do SARS-CoV-2. Em uma pesquisa feita por Cao et al. (2020) da qual participaram 37 profissionais da saúde que atuam junto a pacientes com COVID-19 52,6\% dos enfermeiros apresentaram sentimentos negativos como medo e estresse, o medo está relacionado ao contágio, já o estresse é relativo ao excesso de trabalho. No estudo de Chung e Yeung (2020) feito com 69 profissionais da saúde atuantes no combate ao SARS-CoV-2, 34,8\% dos participantes apresentaram sintomas leves de depressão e 14,4\% relataram sintomas moderados.

O afastamento de profissionais da saúde com mais de 60 anos e daqueles que apresentam doenças crônicas é aconselhado por instituições internacionais e nacionais. Entretanto, em muitos estabelecimentos de saúde brasileiros os mesmos continuam trabalhando. Apesar de a doença trazer mais riscos para aqueles que fazem parte de grupos de risco o número de óbitos entre profissionais com menos de 60 anos e sem comorbidades também causa apreensão (BRASIL, 2020d; 2020h; 2020i; GLYNN, 2020; WHO, 2020e).

É de suma importância a proteção da integridade física e emocional do profissional da saúde. Trabalhadores de estabelecimentos de saúde arriscam suas vidas diariamente para preservar a vida do outro, é preciso que eles tenham seus processos de trabalho valorizados, através de uma infraestrutura adequada, sentindo-se assim protegidos e motivados para continuarem realizando suas funções num momento tão trágico como esse pelo qual a humanidade está passando. Faz-se necessário que instâncias governamentais tenham essas questões como prioridade frente ao contexto da pandemia.

\section{CONCLUSÕES}

A escassez de profissionais da saúde implica no controle da pandemia de COVID-19. O afastamento de profissionais da saúde devido ao contágio pelo SARS-CoV-2 além de diminuir a força de trabalho nas instituições de saúde traz consequências negativas para aqueles que continuam na batalha. Chegará um momento em que a desmotivação dos profissionais da saúde tornará impossível a manutenção da qualidade da assistência. Os profissionais da saúde precisam sentir-se seguros no desenvolvimento de suas funções e apoiados tanto pela administração dos locais de trabalho quanto pelo governo e pela população.

A COVID-19 é uma doença muito perversa, com rápido contágio, resultando em morte para muitos pacientes. Distanciamento e isolamento sociais são indicados pelas autoridades de saúde. Entretanto, para os profissionais da saúde essas medidas não são possíveis, para eles são instituídas outras formas de prevenção, como higienização adequada e utilização correta de equipamentos de proteção, por isso é inaceitável que o SARS-CoV-2 por escassez de EPIs, falta de treinamento de 
pessoal e inexistência de apoio psicossocial continue interrompendo a vida dos profissionais da saúde.

Sem profissionais da saúde não há história, não há vida.

\section{REFERÊNCIAS}

ADAMS, J. G; WALLS, R. M. Supporting the health care workforce during the COVID-19 global epidemic. JAMAS, v. 323, n. 15, p. 1439-1440, 2020.

ALICI, N. S; BEYAN, A. C; SIMSEK, C. Meslek hastaligi olarak COVID-19. Eurasia Journal of Pulmonology. Coronavirus hastaligi 2019 (COVID-19) ve akciger, gogus hastaliklari uzmanlarinin bilmesi gerekenler (Bölüm II), p. 148-165, 2020.

AYDOGDU, A.L.F. Violence and discrimination against healthcare workers in times of new coronavirus. J. nurs. health. v. 10, n. esp., p. e20104006, 2020.

BRASIL. Conselho Federal de Enfermagem - COFEN. Brasil perdeu mais profissionais da enfermagem que Itália e Espanha juntas. Brasília: COFEN, 2020d. Disponível em: https://bit.ly/2ZIV7RM. Acesso em: 07 mai. 2020.

BRASIL. Conselho Federal de Enfermagem - COFEN. Brasil tem 30 mortes na Enfermagem por Covid-19 e 4 mil profissionais afastados. Brasília: COFEN, 2020i. Disponível em: https://bit.ly/2E3YxqB. Acesso em: 04 mai. 2020.

BRASIL. Conselho Federal de Enfermagem - COFEN. Brasil ultrapassa EUA em mortes de profissionais da Enfermagem por Covid-19. Brasília: COFEN; 2020e. Disponível em: https://bit.ly/ 2ZDssxa. Acesso em: 07 mai. 2020.

BRASIL. Conselho Federal de Medicina - CFM. Posição do Conselho Federal de Medicina sobre a pandemia de COVID-19: contexto, análise de medidas e recomendações. Brasília: CFM, $2020 \mathrm{~h}$. Disponível em: https://bit.ly/3mq4Nu4. Acesso em: 06 mai. 2020.

BRASIL. Ministério da Saúde. Alunos da área da saúde poderão ajudar no combate ao coronavírus. Brasília: Ministério da Saúde; 2020g. Disponível em: https://bit.ly/3kiVytN. Acesso em 04 mai. 2020.

BRASIL. Ministério da Saúde. Cerca de 5 milhões de profissionais da saúde serão cadastrados. Brasília: Ministério da Saúde; 2020c. Disponível em: https://bit.ly/2ZFk0xr>. Acesso em: 03 mai. 2020. 
BRASIL. Ministério da Saúde. Ministério da Saúde envia primeiros profissionais voluntários para Manaus. Brasília: Ministério da Saúde; 2020f. Disponível em: https://bit.ly/2RrIHsX. Acesso em: 04 mai. 2020.

BRASIL. Ministério da Saúde. Painel do coronavírus. Brasília: Ministério da Saúde, 2020a. Disponível em: https://covid.saude.gov.br/. Acesso em: 09 de mai. 2020.

BRASIL. Ministério da Saúde. Voluntários podem ajudar contra o coronavirus. Brasília: Ministério da Saúde; 2020b. Disponível em: https://bit.ly/2RuYKGw. Acesso em: 03 mai. 2020.

CAO, J. et al. A study of basic needs and psychological wellbeing of medical workers in the fever clinic of a tertiary general hospital in Beijing during the COVID-19 outbreak. Psychotherapy and Psychosomatics, 2020.

CARLESI, K. C. et al. Patient safety incidents and nursing. Rev Lat Am Enfermagem, v. 25, e2841, 2017.

CETINTEPE, S; ILHAN, M. COVID-19 salgininda saglik çalisanlarinda risk azaltilmasi. Journal of Biotechnology and Strategic Health Research, v. 4, p. 50-54, 2020.

CHA, A. E. More than 9,000 U.S. health-care workers have been infected with the coronavirus. The Washington Post, 14 abr. 2020. Disponível em: https://wapo.st/3mlJmu6. Acesso em: 27 de abr. 2020.

CHUNG, J.P.Y., YEUNG, W.S. Staff mental health self-assessment during the COVID-19 outbreak. East Asian Arch Psychiatry, v.30, n. 1, p. 34, 2020.

DALRI, R. C. M. B; ROBAZZI, M. L. C. C; SILVA, L. A. Riscos ocupacionais e alterações de saúde entre trabalhadores de enfermagem brasileiros de unidades de urgência e emergência. Ciencia $\mathbf{y}$ Enfermeria, v. XVI, n. 2, p. 69-81, 2010.

DIKMEN, A. U. et al. Ankara'da bir universite hastanesinde çalisan saglik personelinin geçirdigini ifade ettigi is kazalarının degerlendirilmesi. Turk Tabipleri Birligi Mesleki Saglik ve Guvenlik Dergisi, v. 14, n. 53, p. 22-29, 2014.

FERNANDES, J. C. Et al. Jornada de trabalho e comportamentos de saúde entre enfermeiros de hospitais públicos. Rev Lat Am Enfermagem, v. 21, n. 5, [08 telas], 2013. 
FIOCRUZ - Fundação Oswaldo Cruz - Pesquisa inédita traça perfil da enfermagem no Brasil. Rio de Janeiro: Fiocruz, 2015. Disponível em: https://bit.ly/33uLiI9. Acesso em: 30 de abr. 2020.

FOLHAPRESS. País tem mais de 8 mil profissionais da saúde afastados em meio à pandemia de coronavírus. Nes Total, 17 abr. 2020. Disponível em: https://bit.ly/35AVyBn. Acesso em: 06 de mai. 2020.

GALLASCH, C. H. Prevenção relacionada à exposição ocupacional do profissional da saúde no cenário de COVID-19. Revista Enfermagem Uerj, v. 28, p. e49596, 2020.

GLYNN, J. R. Protecting workers aged 60-69 years from COVID-19. Lancet, 2020.

GODDERIS, L; BOONE, A; BAKUSIC, J. COVID-19: a new work-related disease threatening healthcare workers. Occup Med (Lond) May 2, kqaa056, 2020. [Epub ahead of print].

GONZAGA, V. Profissionais da saúde representam 36\% dos casos de covid-19 em Pernambuco. Brasil de Fato, 23 abr. 2020. Disponível em: https://bit.ly/3mouVFF. Acesso em: 27 de abr. 2020.

GOULART, A. C. Revisitando a espanhola: a gripe pandêmica de 1918 no Rio de Janeiro. Hist. cienc. saude-Manguinhos, v. 12, n. 1, p. 101-142, 2005.

GRECO, D. B; NEVES, M. C. O profissional da saúde infectado pelo HIV - direitos e deveres. Revista Bioética, v. 1, n. 1, 1993.

HUANG, Y; ZHAO, N. Chinese mental health burden during the COVID-19 pandemic. Asian J Psychiatr, v. 14, n. 51, p. 102052, 2020. [Epub ahead of print].

IBGE - Instituto Brasileiro de Geografia e Estatística. Síntese de indicadores sociais: Uma análise das condições de vida da população brasileira 2018. Estudos \& pesquisas, Informação demográfica e socio-econômica 39. Rio de Janeiro: IBGE, 2018. Disponível em: https://bit.ly/3kkKkF1. Acesso em: 29 de abr. 2020.

ICN - International Council of Nurses - ICN calls for data on healthcare worker infection rates and deaths. Geneva: ICN, 2020. Disponível em: https://bit.ly/3bZC4Y6. Acesso em: 30 de abr. 2020. 
JI, D. et al. Prevalence of psychological symptoms among Ebola survivors and healthcare workers during the 2014-2015 Ebola outbreak in Sierra Leone: a cross-sectional study. Oncotarget, v. 8 n. 8 , p. 12784-12791, 2017.

JOHNS HOPKINS UNIVERSITY. COVID-19 Dashboard by the Center for Systems Science and Engineering (CSSE). Maryland: Johns Hopkins University, 2020. Disponível em: https://coronavirus. jhu.edu/map.html. Acesso em: 09 de mai. 2020.

JOSEPH, B; JOSEPH, M. The health of the healthcare workers. Indian J Occup Environ Med, v. 20, n. 2, p. 71-72, 2016.

LANCET, T. COVID-19: protecting health-care workers. Lancet, v. 395, n. 10228, p. 922, 2020.

MELGAREJO, L. M; BALLÉN, M. N. R. Profesionales de enfermeria y cuidado en las condiciones laborales actuales (Nursing professionals and care of the current labor conditions). Investigacion en Enfermeria: Imagen y Desarrollo, v. 12, n. 2, p. 55-92, 2010.

MENON, V; PADHY, S. K. Ethical dilemmas faced by health care workers during COVID-19 pandemic: Issues, implications and suggestions. Asian J Psychiatr, v. 28 n. 51, p. 102116, 2020. [Epub ahead of print].

NDEJJO, R. et al. Occupational health hazards among healthcare workers in Kampala, Uganda. Journal of Environmental and Public Health, 2015.

NEVES, H. C. C. et al. Segurança dos trabalhadores de enfermagem e fatores determinantes para adesão aos equipamentos de proteção individual. Rev. Latino-Am. Enfermagem, v. 19, n. 2, [08 telas], 2011.

NIA, H. S; LEHTO, R. H; EBADI, A; PEYROVI, H. Death anxiety among nurses and health care professionals: A review article. Int J Community Based Nurs Midwifery, v. 4, n. 1, p. 2-10, 2016.

NUGENT, C. “It's like being a war medic." A Madrid doctor speaks out about grave shortages in protective gear. Time, 02 abr. 2020. Disponível em: https://bit.ly/2ZH8vFY. Acesso em: 27 de abr. 2020.

OECD - Organisation for Economic Co-operation and Development. Health at a Glance 2019: OECD Indicators. Paris: OECD, 2019. 
OIT - Organización Internacional del Trabajo. Cinco formas de proteger al personal de salud durante la crisis del COVID-19. Geneva: OIT, 2020 Disponível em: https://bit.ly/2RwgVeF. Acesso em: 06 mai. 2020.

PORTUGAL. Covid-19/Reforço de recursos humanos. Lisboa: Serviço Nacional de Saúde; 2020. Disponível em: https://bit.ly/2FBFAMq. Acesso em: 06 de mai. 2020.

REGULY, E. Italian doctors' fatalities reach tragic levels as they fight COVID-19 in overburdened hospitals. The Globe and Mail, 03 abr. 2020. Disponível em: https://tgam.ca/33AyEqY. Acesso em: 27 de abr. 2020.

REZENDE, J. M. À sombra do plátano: crônicas de história da medicina [online]. São Paulo: Editora Unifesp, 2009. As grandes epidemias da história. p. 73-82. ISBN 978-85-61673-63-5.

RIO DE JANEIRO. Prefeitura Municipal do. Prefeitura contrata profissionais da saúde para enfrentamento da Covid-19. Rio de Janeiro: Prefeitura do Rio, 2020. Disponível em: https://bit.ly/ 2FGfnfo. Acesso em: 05 mai. 2020.

RODRIGUES, N. H; SILVA, L. G. A. Gestão da pandemia coronavírus em um hospital: relato de experiência profissional. J. nurs. Health, v. 10, n. esp., p. e20104004, 2020.

SANGOI, T. P; GEHLEN, M. H; STOBÄUS, C. D. Riscos ocupacionais para trabalhadores da enfermagem em oncologia: Uma revisão narrativa. Disciplinarum Scientia. Série: Ciências da Saúde, Santa Maria, v. 17, n. 2, p. 301-317, 2016.

SATUR, D. Argentina, en el podio mundial en proporción de personal de salud infectado con Covid-19. La Izquierda Diario, 17 abr. 2020. Disponível em: https://bit.ly/3c2iASJ. Acesso em: 27 de abr. 2020.

SCHWARTZ, J; KING, C. C; YEN, M.Y. Protecting health care workers during the COVID-19 coronavirus outbreak-lessons from Taiwan's SARS response. Oxford University, 2020. Available from: https://bit.ly/3kpkYFZ. Acesso em: 28 de abr. 2020.

SEPKOWITZ, K. A; EISENBERG, L. Occupational deaths among healthcare workers. Emerg Infect Dis, v. 11, n. 7, p. 1003-8, 2005. 
SOUZA E SOUZA, L.P.S; SOUZA, A.G. Enfermagem brasileira na linha de frente contra o novo Coronavírus: quem cuidará de quem cuida? J. nurs. Health, v. 10, n. esp., p.e20104005, 2020.

TURQUIA. Anadolu Ajansi. Saglik çalisan isinden ayrilmasina izin verilmeyecek. Ankara: Anadolu Ajans1, 2020. Disponível em: https://bit.ly/2ZARcGz. Acesso em: 27 de abr. 2020.

ULU, G. "1 hekim oldu, 111 saglikci COVID-19 hastasi oldu." Sozcu, 07 abr. 2020. Disponível em: https://bit.ly/2E31D0q. Acesso em: 27 de abr. 2020.

VELLOSO, M. P. Os restos na história: percepções sobre resíduos. Ciênc. saúde coletiva, v. 3, n. 6, p. 1953:1964, 2008.

WANG, J; ZHOU, M; LIU, F. Exploring the reasons for healthcare workers infected with novel coronavirus disease 2019 (COVID-19) in China. Journal of Hospital Infection, pre-proof., 2020.

WHO - World Health Organization . Coronavirus disease (COVID-19) outbreak: rights, roles and responsabilities of health workers, including key considerations for occupational safety and health. Interim guidance. Geneva: WHO, 2020e Disponível em: https://bit.ly/33vFQ7Z. Acesso em: 03 mai. 2020.

WHO - World Health Organization. Mental health and psychosocial considerations during the COVID-19 outbreak. Geneva: WHO; 2020d. Disponível em: https://bit.ly/2Fzl4ff. Acesso em: 02 mai. 2020.

WHO - World Health Organization. Novel Coronavirus - China. Geneva: WHO, 2020. Disponível em: https://bit.ly/35G5REk. Acesso em: 28 abr. 2020.

WHO - World Health Organization. Shortage of personal protective equipment endangering health workers worldwide. Geneva: WHO, 2020b. Disponível em: https://bit.ly/3mqL6Cs. Acesso em: 30 abr. 2020.

WHO - World Health Organization. Rational use of personal protective equipment for coronavirus disease (COVID-19) and considerations during severe shortages. Geneva: WHO, 2020c. Disponível em: https://bit.ly/2MHNnMa. Acesso em: 30 abr. 2020. 
WYZGOWSKI, P. et al. Occupational HIV risk for health care workers: risk factor and the risk of infection in the course of professional activities. The Clin Risk Manag, v. 12, p. 989-994, 2016.

YAZICI, G; ELBAS, N. O. Hemsirelerin hasta ve hemsire guvenligi açisindan acil servislerin çalısma ortamina iliskin gorusleri (Conceptions of nurses concerning safety of patient and nurse in emergency service working environment). Sag Aka Derg (Health Care Acad J), v.4, n.2, p. 69-80, 2017.

ZHOU, X. et al. Burnout, psychological morbity, job stres, and job satisfaction in Chinese neurologists. Neurology Journals 2017 88(18): 1727-1735, 2017. 
